\title{
Conservative management of a bladder leiomyosarcoma in a 43-year-old patient
}

\author{
Aikaterini Anastasiou, Ioannis Katafigiotis, Spyridon Skoufias, Ioannis Anastasiou, \\ Constantinos Constantinides \\ $1^{\text {st }}$ University Urology Clinic, Laiko Hospital, Athens, Greece.
}

\begin{abstract}
Summary Leiomyosarcoma of the bladder is an aggressive and rare tumor, with less than

200 reported cases. The treatment of bladder leiomyosarcoma is controversial although in most cases an aggressive surgical therapy is preferred. Usually, a radical cystectomy is performed, as it is considered to have a better disease-specific survival rate. A 43-year-old man presented to our Urology Department with painless macroscopic hematuria.

He was submitted to transurethral resection of the tumor. The transurethral resection was complete and revealed only this small single lesion and the rest of the bladder was normal with no other lesion or suspicious lesion. The final histology revealed leiomyosarcoma of the bladder. Due to his age and the aggressiveness of the tumor after a thorough and detailed discussion with the patient a conservative management with aggressive follow up was decided. The patient a year after the diagnosis is in perfect condition without sign of a recurrence or metastastes.
\end{abstract}

KEY WORDS: Bladder leiomyosarcoma; Bladder carcinoma; Rare bladder tumor; Non urothelial bladder cancer.

Submitted 13 October 2017; Accepted 19 November 2017

\section{INTRODUCTION}

Leiomyosarcoma of the bladder is an aggressive and rare tumor, with less than 200 reported cases (1). It relates to $1 \%$ of all bladder malignancies (1). Patients have a median age of 65 years, at diagnosis (2), with no clear evidence of any sex prevalence. The prognosis of the tumor is poor, with an approximately 46 months survival (1). We present a case of a 43-year-old man with leiomyosarcoma of the bladder.

\section{Case report}

A 43-year-old man presented to our Urology Department (1 $1^{\text {st }}$ University Urology Clinic, Laiko Hospital) with painless macroscopic hematuria. He had no other symptom and no other co morbidities. The patient was a heavy smoker, but he did not have any other risk factors.

The ultrasound of the bladder was normal and the rest of the general examinations of the blood and urine were normal apart from the presence of blood in the urine.
The urine cytology was negative for malignancy. He was submitted to flexible cystoscopy, during which a single small exophytic lesion in the left lateral bladder wall, was revealed. The patient also underwent computed tomography (CT), which was negative for metastases. He was submitted to transurethral resection of the tumor. The transurethral resection was complete and revealed only this small single lesion and the rest of the bladder was normal with no other lesion or suspicious lesion. The final histology revealed leiomyosarcoma of the bladder (Figure 1). Due to his age and the aggressiveness of the tumor after a thorough and detailed discussion with the patient a conservative management with aggressive follow up was decided. One month after the surgery, a new biopsy from the scar of the previous operation and random biopsies were also performed, all of which were negative. Three months postoperatively the cystoscopy was negative for recurrence. He was submitted to a third cystoscopy with random biopsies after six months again with negative results for malignancy (10 months after the first operation). The patient a year after the diagnosis is in perfect condition without sign of a recurrence or metastastes. The next cystoscopy and CT tomography of the abdomen are planned in six months.

\section{Figure 1.}

Neoplastic spindle cells with concomitant neo-vascular angiogenesis and sparks lymphocytic presence.

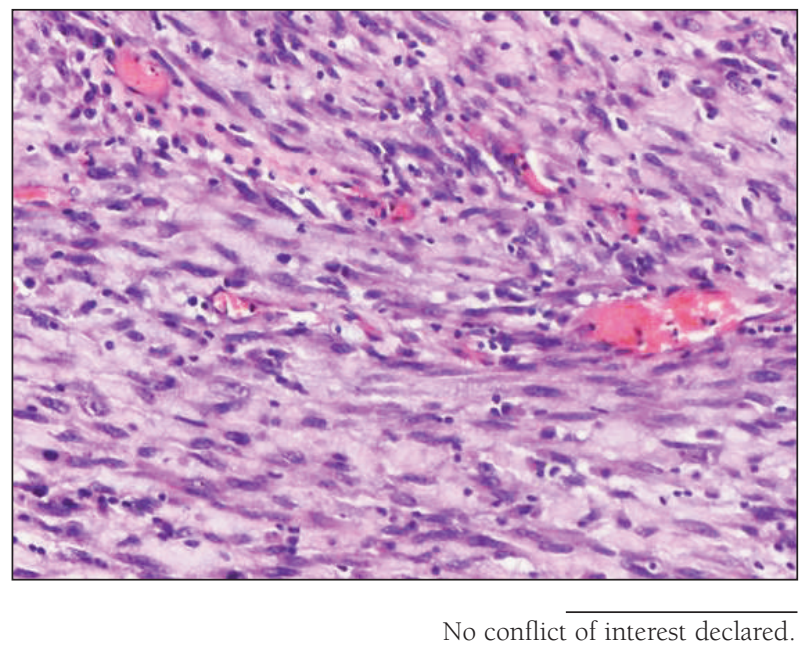




\section{Discussion}

Leiomyosarcoma of the bladder is a very rare type of bladder tumor. The annual age-adjusted incidence rate is only 0.23 cases per $1,000,000$ and there is no significant change over time (2). It is considered to be the most common bladder sarcoma, however it only accounts for $0.1 \%$ of all non-urothelial tumors (2). Different risk factors as the use of cyclophosphamide and ketamine may lead to the development of the disease (1). Furthermore, the exposure to local pelvic radiotherapy or systemic chemotherapy and the use of tobacco seems to be the most common etiological factors, due to genetic alterations (1). Our patient was a heavy smoker without any other co-morbidity. The pathophysiology of bladder leiomyosarcoma is not clear. The main symptoms are gross hematuria, dysuria, urinary frequency and abdominal pain (2). Hematuria is the most common symptom, and in our case, was the main and only symptom. The treatment of bladder leiomyosarcoma is controversial although in most cases an aggressive surgical therapy is preferred. Usually, a radical cystectomy is performed, as it is considered to have a better disease-specific survival rate $(1,2)$. Other treatment modalities that have been suggested are the partial cystectomy, the transurethral resection of a bladder tumor (TURBT) for small local tumors usually as a part of a multimodality treatment, or radiotherapy (2). Neoadjuvant or adjuvant chemotherapy is also applied (2). Neoadjuvant chemotherapy is used in patients with locally advanced disease. The most common neoadjuvant regimens are doxorubicin, ifosfamide, cisplatin, adriamycin, and vincristine with favorable outcomes (1). For the treatment of smaller leiomyosarcomas $(<4 \mathrm{~cm})$ a partial cystectomy is preferred, as it provides therapeutic efficacy, but also a better quality of life to the patient, compared to more radical surgeries $(1,3)$. In our case, considering the young age of our patient (43 years old), after a thorough discussion a conservative management avoiding aggressive surgical methods but combined with a close follow-up was decided. Finally, there is no consensus regarding the prognosis of the disease or the follow-up of the patients. Patients with bladder leiomyosarcomas usually have a very poor prognosis with a median survival time of 46 months, with a survival rate of $62 \%$ in 5 years $(1,3)$. Important prognostic factors are the presence of tumorfree margins, local invasiveness, tumor size and the tumor grade. The poor prognosis of leiomyosarcoma is attributed to the fact that more than $60 \%$ of tumours are aggressive, and lead to metastasis, some of them even in a low stage. The overall local recurrence percentage of the disease is $16 \%$ recurring mainly in the pelvis. Our patient a year after the diagnosis is in perfect condition without sign of a recurrence or metastastes.

\section{Conclusion}

Leiomyosarcoma of the bladder is a rare tumor that is difficult to diagnose and treat. The disease is considered to be highly aggressive. As a result a radical treatment, with detrimental effects to the quality of life of the patient is usually considered as necessary. However, a more conservative management of the patient should be considered, when treating a young person especially with small solitary lesions, but with a strict follow-up. Prognosis is generally poor.

\section{REFERENCES}

1. Fakhoury M, Hwang RR, Silletti J, Bjurlin MA. Bladder leiomyosarcoma: A rare, but aggressive diagnosis. Curr Urol. 2016; 9:166-168

2. Rodríguez D, Preston MA, Barrisford GW, et al. Clinical features of leiomyosarcoma of the urinary bladder: analysis of 183 cases. Urol Oncol. 2014; 32:958-65.

3. Slaoui H, Sanchez-Salas R, Validire P, et al. Urinary bladder leiomyosarcoma: primary surgical treatment. Urol Case Rep. 2014; 2:137-8.

\author{
Correspondence \\ Aikaterini Anastasiou, MD (Corresponding Author) \\ aikatianast@gmail.com \\ Ioannis Katafigiotis, MD \\ katafigiotis.giannis@gmail.com \\ Spyridon Skoufias, MD \\ spyskouf@hotmail.com \\ Ioannis Anastasiou, MD \\ ekati2@otenet.gr \\ Constantinos Constantinides, MD \\ ckonstan@med.uoa.gr \\ $1^{\text {st }}$ University Urology Clinic, Laiko Hospital \\ Agiou Thoma 17, Athens 11527, Greece
}

